Forum 2018 · 33:218

https://doi.org/10.1007/s12312-018-0441-0

Online publiziert: 4. Juli 2018

(c) Springer Medizin Verlag GmbH, ein Teil von

Springer Nature 2018

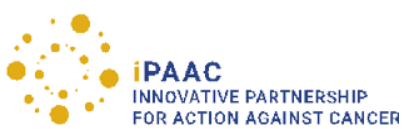

Am 16. und 17. April 2018 fiel in Luxemburg der Startschuss für das neue EU-Projekt „iPAAC - innovative Partnership for Action Against Cancer". In den kommenden drei Jahren werden insgesamt 24 Partner und 40 assoziierte Partner aus 24 europäischen Ländern zusammen daran arbeiten, innovative Ansätze zur Krebsbekämpfung umzusetzen, aufbauend auf den Ergebnissen der vorangegangenen Joint Action CANCON (Cancer Control).

Die Entwicklung einer sogenannten Roadmap, die die nachhaltige Umsetzung von Maßnahmen zur Bekämpfung von Krebs sowie deren praktische Anwendungen darstellt, werden die wichtigsten Ergebnisse der Joint Action sein.

Die Joint Action besteht aus insgesamt 10 Arbeitspaketen, die unter anderem die Krebsprävention und die Entwicklung von ganzheitlichen Konzepten zur Integration der Erkenntnisse aus der Genomforschung zum Ziel hat. Weitere Schwerpunkte liegen auf dem Ausbau und der Vernetzung der europäischen Krebsregister und dem Mapping von innovativen Krebsbehandlungen. Die Verbesserung der Steuerung und des Managements der Krebsversorgung inklusive einer Aktualisierung der Nationalen Krebspläne sind weitere Arbeitspakete der Joint Action.

\title{
Startschuss für die neue Joint Action iPAAC
}

\section{Das „Arbeitspaket" der Deutschen Krebsgesellschaft}

Im Rahmen des von der Deutschen Krebsgesellschaft geleiteten Arbeitspaketes 10 „Governance of Integrated and Comprehensive Cancer Care" sollen praktische Instrumente (weiter-)entwickelt werden, die eine standardisierte tumorspezifische und alle Bereiche umfassende onkologische Versorgung in allen europäischen Mitgliedstaaten gewährleisten und den Patienten einen niederschwelligen Zugang zu qualitätsgesicherten Behandlungen ermöglichen.

Dieses Ziel soll durch die Umsetzung folgender Aufgaben erreicht werden:

1. Überprüfung der nationalen Krebspläne (NCCP) und Entwicklung von Empfehlungen zu deren Aktualisierungen (Leitung: Nationales Institut für öffentliche Gesundheit, Slowenien)

2. Entwicklung eines generischen onkologischen Patientenpfades, der im Rahmen des Comprehensive Cancer Care Network (CCCNs) pilotiert und implementiert wird (Leitung: Health-ICT-Team, Fakultät für Wirtschaftswissenschaften, TU Dresden, Deutschland)

3. Entwicklung einer standardisierten Methodik zur Ableitung von Qualitätsindikatoren und darauf basierend die Ableitung von einem Set tumorspezifischer Qualitätsindikatoren für die Nutzung in den CCCNs (Leitung:
Abteilung für öffentliche Gesundheit und Infektionskrankheiten, Sapienza Universität, Italien)

4. Entwicklung eines Frameworks für die Umsetzung und Pilotierung von Patienten berichteten Ergebnissen (PROs, patient reported outcomes) in den CCCNs (Leitung: Zentrum für evidenzbasierte Gesundheitsversorgung ZEGV, TU Dresden in Zusammenarbeit mit der Deutschen Krebsgesellschaft)

5. Entwicklung von generischen und tumorspezifischen Anforderungen für den Aufbau von CCCNs, die Entwicklung eines Frameworks zum Monitoring der erfolgreichen Umsetzung der Anforderungen sowie die Implementierung von zwei Pilot-Comprehensive-Cancer-Care-Networks (Leitung: Deutsche Krebsgesellschaft, Bereich Zertifizierung)

Die Auftaktveranstaltung für das Arbeitspaket 10 fand vom 7. bis 8. Mai 2018 in der Geschäftsstelle der Deutschen Krebsgesellschaft in Berlin statt. Bis zum zweiten Treffen im Februar 2019 sollen bereits erste Ergebnisse vorliegen. Weitere Information zur Joint Action iPAAC finden Sie unter: http://ipaac.eu.

\section{Korrespondenzadresse}

Ellen Grießhammer

Deutsche Krebsgesellschaft griesshammer@krebsgesellschaft.de 